\title{
Teaching Methods And Technologies: Aggregated Faculty Analysis, Conclusions And Recommendations Phase IV
}

Dennis L. Payette, (E-mail: payette@adelphi.edu), Adelphi University Daniel Verreault, (E-mail: dverreault@ut.edu), University of Tampa

\begin{abstract}
This paper culminates three years of research on the use of various teaching technologies and methods by the faculty of Adelphi University School of Business in Garden City, New York. Previously, papers on this research were published on the development of the research instrument, the administration and data analysis for full time faculty (Part II), and most recently the analysis of data from adjunct faculty (Part III). This paper (Part IV) includes a number of new faculty additions to the data set and analyzes and interprets the aggregated data. Our overall findings suggest a wide variety of soft and hard technologies where the aggregate group expressed a statistically significant higher perceived "value of use" than a "level of use". Newer classroom types were also valued more highly than used. The research controlled for "department", "status", and "teaching experience". Factors tended to be non-significant with some interesting exceptions. We note our conclusions, make policy recommendations, and suggest opportunities for expanded research.
\end{abstract}

\section{THE CASE STUDY SETTING}

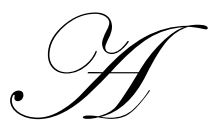

delphi University is a medium sized (8,000 students) private sector university offering bachelor's, master's and doctoral degrees located in Garden City, New York in the United States. This case study was conducted in the School of Business at Adelphi University which offers bachelors and MBA degrees in business. Approximately two thirds of the students are in part and full-time undergraduate programs, and one third enroll in graduate business programs in traditional formats as well as in accelerated cohort formats. Adelphi University is in the final year of candidacy for consideration for accreditation by AACSB-International, The Association to Advance Collegiate Schools of Business. As such it was determined that it would be appropriate to conduct pedagogical research as the institution's mission emphasizes quality teaching as one of its most important features. ${ }^{1}$

\section{RESEARCH METHODOLOGY AND DATA COLLECTION METHODS}

The first problem was to clarify the objective of the research and to define the problem, namely, how would data be collected from faculty on how they actually use and evaluate various teaching methods and technologies. The first procedural step was to make a presentation at a full faculty meeting about the idea of having such research conducted in the School of Business and to ask if the faculty would be willing to participate in the research once the scope and methods were defined. Fortunately, the faculty was highly receptive to the idea. The next step was to conduct a review of the literature on the topic areas to be covered in the research and to try to find an existing suitable

\footnotetext{
${ }^{1}$ The principal author is a member of the full time faculty, chair of the AACSB-I accreditation committee. The co-author of Phase II, III, and IV is former chair of Adelphi's AACSB-I committee, and now teaches at the Sykes College of Business at The University of Tampa.
} 
instrument. It was then determined that a newly designed survey instrument would be the most appropriate method to gather the desired information. An instrument was designed, revised, pre-tested, modified, and finalized. The coding on the instrument is on a 4 point scale for both level of use and the value of use of a particular technology. The response "Intend to use" coded as " 5 " under level of use was used to attempt to discover which technologies were being actively considered by faculty. The response "Have not used" coded as "5" under "value of use" was coded as missing in the analysis. In addition, the status of groups as full-time, adjunct, and new was coded on the mailing. Faculty responded at both the undergraduate and graduate levels for each value and use item. The technologies were classified as "soft" if they were primarily involved with ways of organizing or directing the class, "hard" if the technology was primarily a product whether hardware or software, and "classroom type" addressed the teaching setting (29).

Compared to the previous analyses, teaching experience categories were reduced from four to three as the prior categories did not yield significant differences, and the reduced category count helps populate the cells for analysis. Lastly, the wording of the "value of use" scale in Figure 1 is revised to improve clarity. In terms of identifying "new" faculty, who are in fact full-time, the data collection for the new faculty consisted of a separate survey for all new faculty members.

In Phase II, we updated the literature review, administered the instrument to the full time faculty, and analyzed and reported the results (30).

The next logical step was to survey the adjunct faculty. In Phase III, the authors reviewed the literature on the use of adjunct faculty, including recent changes in AACSB-I guidelines. The results of the pooled groups and tests for differences between groups were reported in (31). The issue of adjunct (part-time, non-participating) faculty is an important issue. Descriptive data about and resources for adjunct faculty is available from several sources. (See $4,10,13,17,19,20,21,23,26,39$, and 40). Evaluative and critical views of the trends in faculty staffing are also available. (See 6, 8, 9, 12, 14, 16, 34, 35, and 43). AACSB-I has recognized the importance of the issue and developed new standards involving what the new standards call non-participating faculty (1). Research that identifies and includes part-time faculty and which can lead to identification of areas in a business school where integration may be improved are needed. This case may serve such a purpose.

As a part of the accreditation initiatives fourteen new full time faculty members were hired during the two years after the initial survey of full time faculty. Therefore, the same instrument was administered to the fourteen new faculty members to add to the data base, now fifty-five cases. The purpose of this article is to look at the larger data set and to revisit overall patterns, and to examine whether there are significant differences on the factors for which we controlled with special emphasis on the new faculty. The new faculty presumably had not been acculturated and may present a different profile. On the other hand, preferences on the dimensions of technologies and classroom types may be more universal. We may glean preliminary information on this aspect. Most importantly, our goal is to develop a profile of how the faculty use and value the various teaching methods and technologies. We believe that this data can help administrators and faculty members identify opportunities to close the "value and use" gap as well as to help identify similarities and differences across departments and groups.

\section{THE ISSUE OF FACULTY PARTICIPATION}

The American Association of University Professors 1940 Statement of Principles on Academic Freedom and Tenure (36) in section "6" states that:

Teachers are entitled to freedom in the classroom in discussing their subject...with limitations about presenting controversial matter which has no relation to their subject.

The statement does not explicitly address or protect the methods or technologies now available to enhance and or augment the instructional process. Direct observation of teaching or other types of evaluation within the classroom in higher education has generally been regarded as a violation of "academic freedom" unless consented to either by advance agreement such as may be found in collective bargaining documents or by institutional policies 
made clear prior to employment. Therefore, obtaining data from faculty about their teaching methods and procedures cannot generally be required or forced lest claims of unwarranted intrusion or violation of academic freedom is alleged.

Figure 1

The Research Questionnaire

Faculty Survey On Teaching Methods And Technologies

\begin{tabular}{|c|c|c|c|c|c|}
\hline Teaching Status & full-time & part-t & - & & \\
\hline Teaching Experience: & $<4$ & $4-12$ & & $>12$ & \\
\hline Teaching Field: & Acct. & Fin/Eco. & Mkt._ & Mgmt. & MIS/Ops. \\
\hline
\end{tabular}

Scales:

Level of Use:

1. Never

2. Occasionally

3. Frequently

4. Always

5. Intend to use
Value of Use:

1. None or little value

2. Some value

3. Good value

4. Excellent value

5. Have Not Used

Teaching Methods And Technologies With Level Of Use And Value Of Use

\begin{tabular}{|c|c|c|c|c|}
\hline \multirow{2}{*}{$\begin{array}{c}\text { Technologies/ } \\
\text { Methods }\end{array}$} & \multicolumn{2}{|c|}{ Graduate } & \multicolumn{2}{|c|}{ Undergraduate } \\
\hline & Level of Use & Value of Use & Level of Use & Value of Use \\
\hline \multicolumn{5}{|l|}{$\begin{array}{l}\text { Soft Technologies } \\
\end{array}$} \\
\hline & & & & \\
\hline \multicolumn{5}{|l|}{ Essay Examinations } \\
\hline \multicolumn{5}{|l|}{ Attendance } \\
\hline \multicolumn{5}{|l|}{ Research Projects } \\
\hline \multicolumn{5}{|l|}{ Guest Lecturers } \\
\hline \multicolumn{5}{|l|}{ Student Teams } \\
\hline \multicolumn{5}{|l|}{ Other (specify) } \\
\hline \multicolumn{5}{|l|}{ Hard Technologies } \\
\hline \multicolumn{5}{|l|}{ Overhead Projector } \\
\hline \multicolumn{5}{|l|}{ Email } \\
\hline \multicolumn{5}{|l|}{ Streaming Video } \\
\hline \multicolumn{5}{|l|}{ Internet Access } \\
\hline \multicolumn{5}{|l|}{ Blackboard Program } \\
\hline \multicolumn{5}{|l|}{ PowerPoint } \\
\hline \multicolumn{5}{|l|}{ Publisher Aids, e.g. CD’s, VCR, DVD's } \\
\hline \multicolumn{5}{|l|}{ Laser Pointer } \\
\hline \multicolumn{5}{|l|}{ Laptop } \\
\hline \multicolumn{5}{|l|}{ Elmo Projector } \\
\hline \multicolumn{5}{|l|}{ Infocus Projector } \\
\hline \multicolumn{5}{|l|}{ Other (specify) } \\
\hline \multicolumn{5}{|l|}{ "Class Rooms } \\
\hline \multicolumn{5}{|l|}{ Smart Classroom } \\
\hline \multicolumn{5}{|l|}{ Hybrid Classroom } \\
\hline Standard Classroom & & & & \\
\hline
\end{tabular}

Copyright Dennis L. Payette 2006 
It is a basic tenet of this paper that obtaining data about teaching methods and application of modern technologies can be obtained from faculty providing sensitivity to the issues of academic freedom are addressed, anonymity is assured, and potential benefits are explained. This case study illustrates how information was obtained from over fifty faculty members, full time and part time, tenured and non-tenured, on the use of various technologies and teaching methods that they may or may not use in their regular instructional routines. A report on the relational and developmental aspects of research in this setting is forthcoming (32).

\section{FINDINGS}

The sample for this paper included fifty-five cases including full time $(\mathrm{n}=21)$, adjunct $(\mathrm{n}=18)$, and new full time $(\mathrm{n}=16)$. The results were analyzed using SPSS version 12 . The means of the scores for "soft technologies" are presented in Figure 2. Each technology is measured as either undergraduate or graduate, and at each of those a value and a level of use is reported. So for each technology there are four measures. Comparisons can be made between the value and level of use of a given technology at the program level or one may compare value of use or level of use between programs. Comparisons may also be made concerning the rankings of means between technologies to determine the most (or least) highly valued or used technologies. Covering all the comparisons will be impossible in this paper, but we will discuss a variety of reasonable and interesting comparisons.

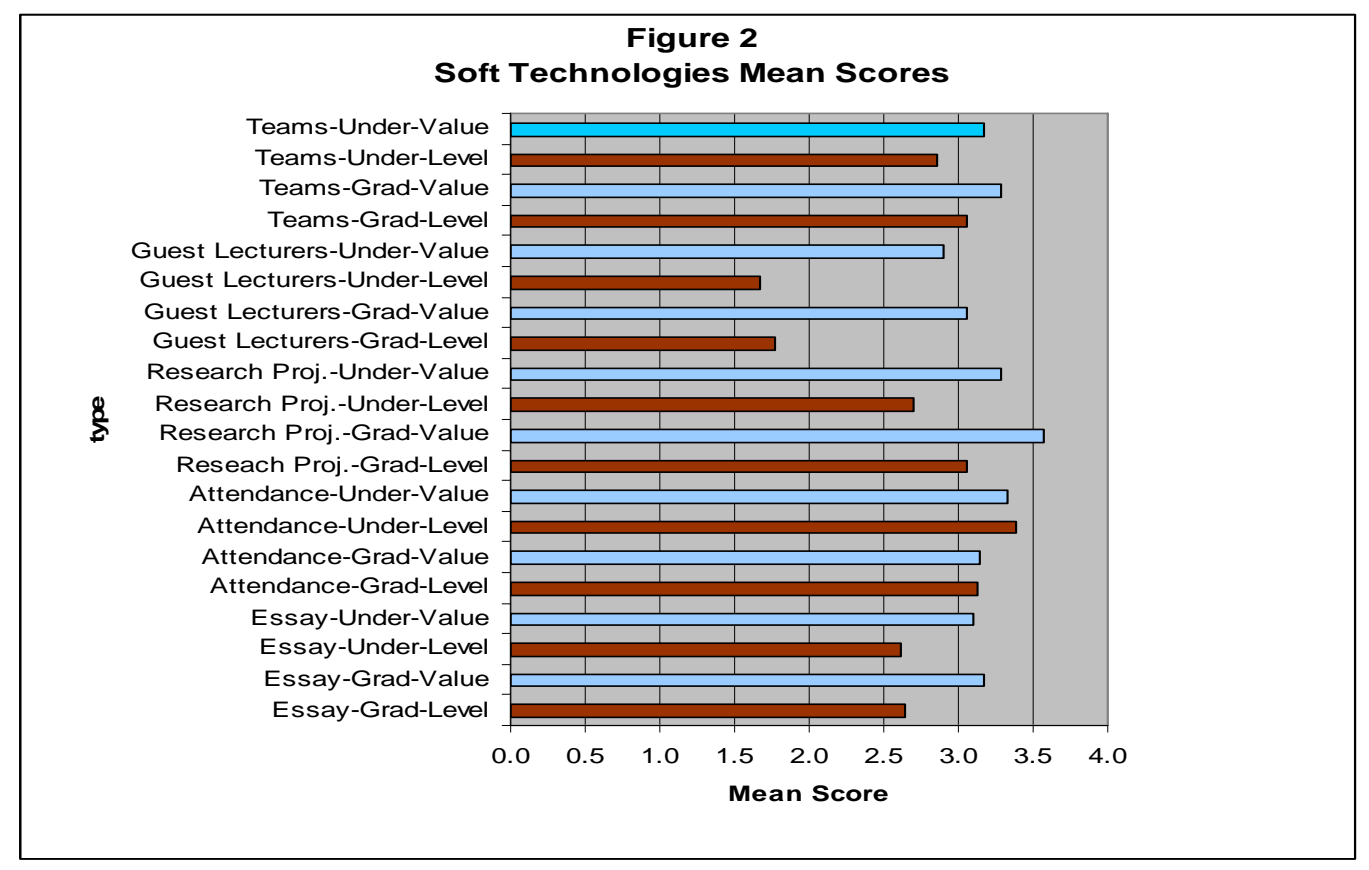

The value of research projects at the graduate level is the highest ranked soft technology, while the use of guest lecturers at the undergraduate level is the lowest ranked mean. Note that the value of guest lecturers is much higher than their level. One may surprise that some constraint causes this gap. Perhaps class time or availability of speakers precludes these variables being more in balance. On the other hand, the four measures concerning attendance seem to be closely aligned at the undergraduate and graduate levels. 


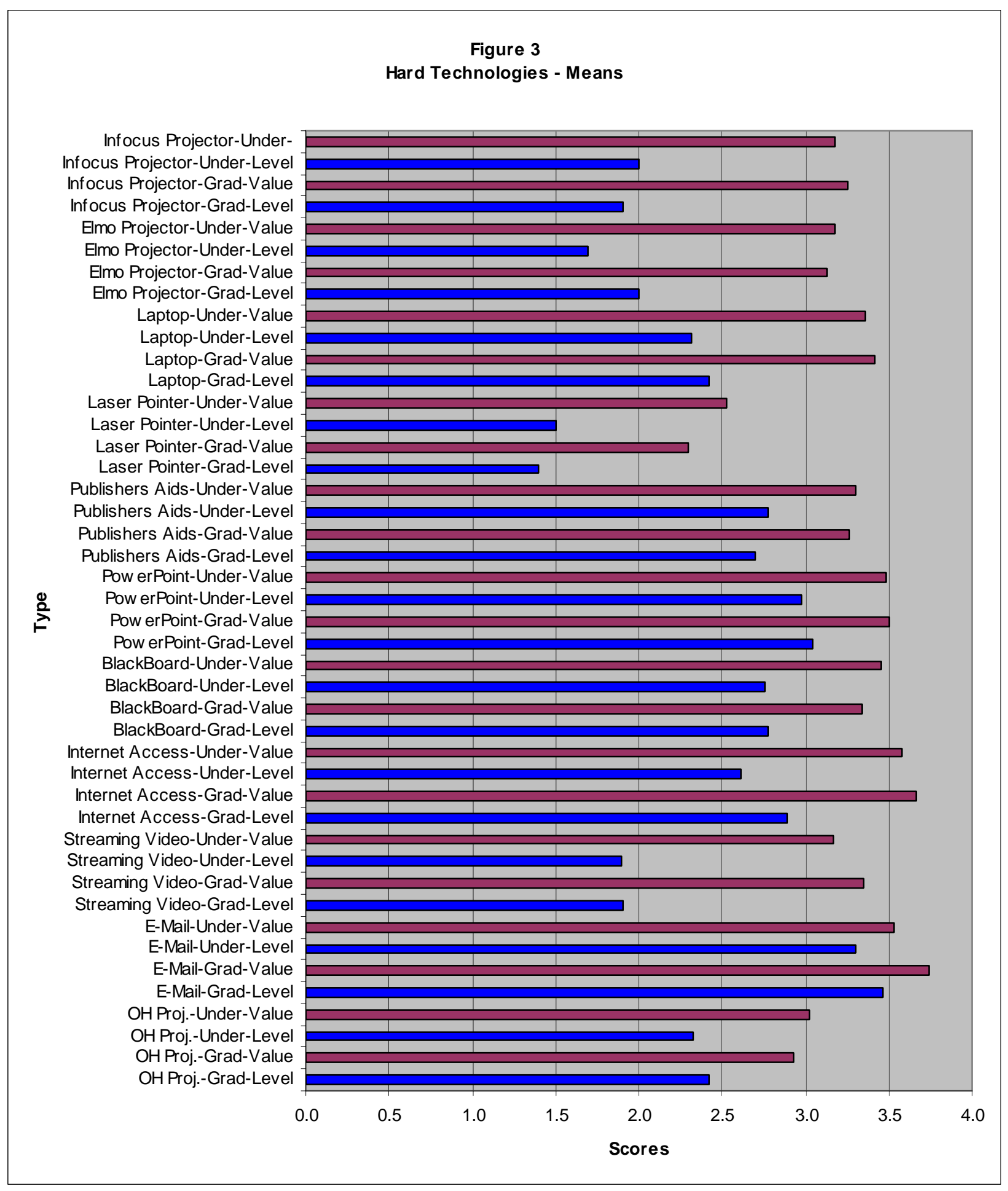


The means for the "hard technologies" are presented in Figure 3. The hard technologies present a visual image of means with greater gaps between value and use than the soft technologies. There is a preliminary impression of the values of many of these technologies exceeding the levels of their use. Again, we think that a fruitful way to approach the gap is through constraint analysis. Some constraints might be physical, some might be related to training, some might involve a level of inertia or resistance to leave one's comfort zone, and some might be related to policy.

The mean scores for the "classroom types" are presented in Figure 4. A visual inspection of the pattern of the mean scores shows that technology equipped classrooms are valued more than used. On the other hand, standard classrooms present the reverse picture. Here, the constraint analogy is clearly pertinent. As the institution builds out more technology equipped classrooms, demand exceeds supply. We surmise that some other technology gaps would close with the elevation of the classroom constraint.

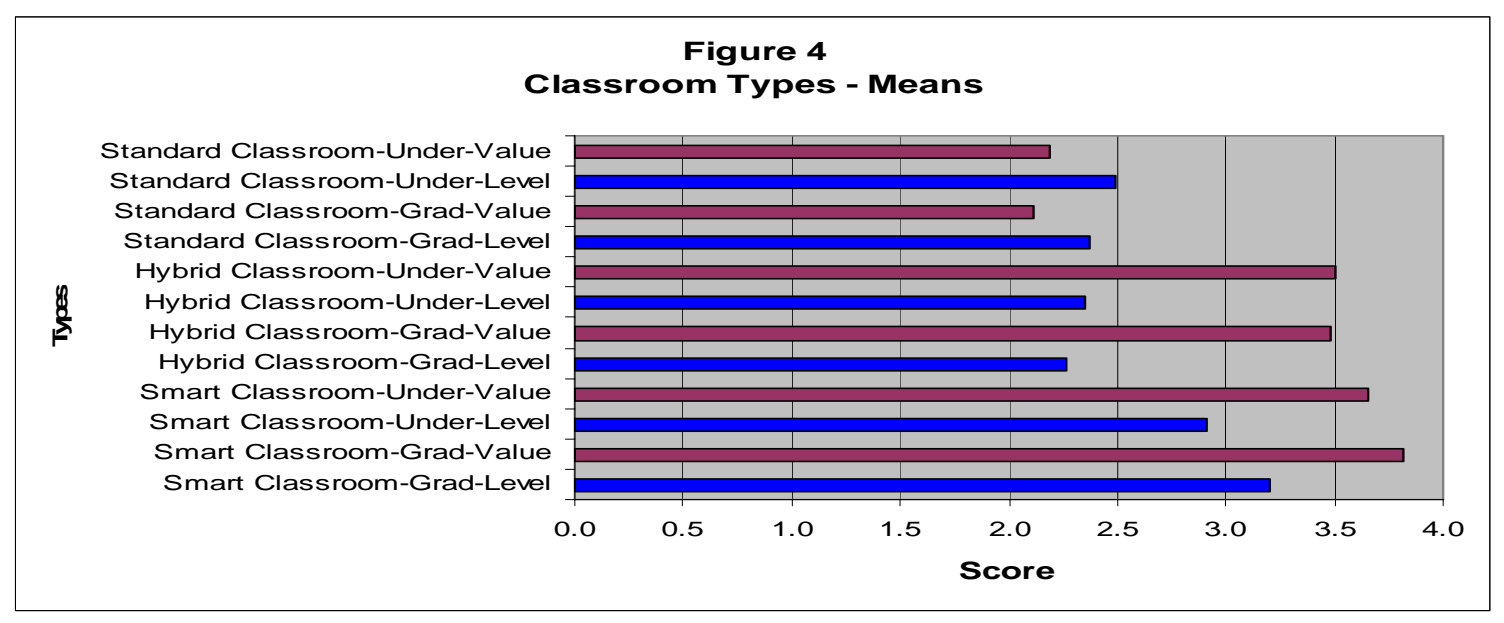

The means of the highest and lowest scoring measures are presented in Table 1. The list of highest scoring means is dominated by value of use variables. A significant exception is e-mail and attendance. The use of both of email and attendance face little or no constraint so the levels of both are ranked highly along with their values. The lowest scoring means are dominated by the more esoteric or infrequently encountered hard technologies. Some of these may be contingent upon other constraints such as technology equipped classrooms. Guest lecturers at both the graduate and undergraduate levels both appear as little used. 
Table 1

Highest And Lowest Scoring Measures

Panel A: Scores Greater Than 3.2

\begin{tabular}{|c|c|c|}
\hline Type & Measure & 3.4 \\
\hline Soft & Attendance-Under-Level & 3.3 \\
\hline Soft & Attendance-Under-Value & 3.3 \\
\hline Soft & Research Proj-Under-Value & 3.3 \\
\hline Soft & Teams-Grad-Value & 3.5 \\
\hline Hard & E-Mail-Grad-Level & 3.7 \\
\hline Hard & E-Mail-Grad-Value & 3.3 \\
\hline Hard & E-Mail-Under-Level & 3.3 \\
\hline Hard & E-Mail-Under-Value & 3.6 \\
\hline Hard & Streaming Video-Grad-Value & 3.3 \\
\hline Hard & Internet Access-Under-Value & 3.5 \\
\hline Hard & BlackBoard-Grad-Value & 3.5 \\
\hline Hard & BlackBoard-Under-Value & 3.5 \\
\hline Hard & PowerPoint-Grad-Value & 3.3 \\
\hline Hard & PowerPoint-Under-Value & 3.3 \\
\hline Hard & Publishers Aids-Grad-Value & 3.4 \\
\hline Hard & Publishers Aids-Under-Value & 3.4 \\
\hline Hard & Laptop-Grad-Value & 3.3 \\
\hline Hard & Laptop-Under-Value & 3.8 \\
\hline Hard & Infocus Projector-Grad-Value & 3.7 \\
\hline Class & Smart Classroom-Grad-Value & 3.5 \\
\hline Class & Smart Classroom-Under-Value & 3.5 \\
\hline Class & Hybrid Classroom-Grad-Value & \\
\hline
\end{tabular}

Panel B: Scores Less Than 2.1

\begin{tabular}{|c|c|c|}
\hline Type & Measure & Mean \\
\hline Soft & Guest Lecturers-Grad-Level & 1.8 \\
\hline Soft & Guest Lecturers-Under-Level & 1.7 \\
\hline Hard & Streaming Video-Grad-Level & 1.9 \\
\hline Hard & Streaming Video-Under-Level & 1.4 \\
\hline Hard & Laser Pointer-Grad-Level & 1.5 \\
\hline Hard & Laser Pointer-Under-Level & 2.0 \\
\hline Hard & Elmo Projector-Grad-Level & 1.7 \\
\hline Hard & Elmo Projector-Under-Level & 1.9 \\
\hline Hard & Infocus Projector-Grad-Level & 2.0 \\
\hline Hard & Infocus Projector-Under-Level & \\
\hline
\end{tabular}

We ran paired sample t-tests for combinations of soft technologies and these are presented in Table 2. There may be other combinations of interest. We will describe here just three interesting comparisons as space precludes a full review. We made four comparisons for each technology. The sign of the mean indicates the direction of the difference. For example, for Pair 1 the value of essays at the graduate level was .3333 higher than the level of use of essays and this was significant at .012. A similar interpretation can be applied to Pair 2 as for Pair 1 . Again, one may speculate on the constraint that may cause these differences. Here, the constraint may be one of time rather than technology. A similar interpretation of the finding might be appropriate for Pairs 9 and 10 that compare the levels and value of use of research projects at the undergraduate and graduate programs. Both differences are highly significant and both again illustrate the value-use gap. Since research projects are not technology constrained, a fruitful discussion could involve the factors that cause this gap and what might be done to alleviate it. Pairs 17 through 20 cover the use of teams. Both the levels and value of team use are non-significant, indicating that teams are used at 
about what they are valued. Moreover, teams are both used and valued more in the graduate than the undergraduate program (pairs 19 and 20) are significant.

Table 2

Paired Sample t-Tests

\begin{tabular}{|c|c|c|c|c|c|c|c|}
\hline & & Soft Techr & logies & & & & \\
\hline & & Mean & $\begin{array}{c}\text { Std. } \\
\text { Deviation }\end{array}$ & $\begin{array}{l}\text { Std. Error } \\
\text { Mean }\end{array}$ & $\mathrm{t}$ & $\mathrm{df}$ & $\begin{array}{l}\text { Sig. (2- } \\
\text { tailed) }\end{array}$ \\
\hline Pair 1 & $\begin{array}{l}\text { Essay-Grad-Level } \\
\text { Essay-Grad-Value }\end{array}$ & -.33333 & .81650 & .12599 & -2.646 & 41 & .012 \\
\hline Pair 2 & $\begin{array}{l}\text { Essay-Under-Level } \\
\text { Essay-Under-Value }\end{array}$ & -.33333 & 1.00406 & .15493 & -2.152 & 41 & .037 \\
\hline Pair 3 & $\begin{array}{l}\text { Essay-Grad-Level } \\
\text { Essay-Under-Level }\end{array}$ & .16216 & .89795 & .14762 & 1.099 & 36 & .279 \\
\hline Pair 4 & $\begin{array}{l}\text { Essay-Grad-Value } \\
\text { Essay-Under-Value }\end{array}$ & .30303 & .76994 & .13403 & 2.261 & 32 & .031 \\
\hline Pair 5 & $\begin{array}{l}\text { Attendance-Grad-Level } \\
\text { Attendance-Grad-Value }\end{array}$ & .24390 & .73418 & .11466 & 2.127 & 40 & .040 \\
\hline Pair 6 & $\begin{array}{l}\text { Attendance-Under-Level } \\
\text { Attendance-Under-Value }\end{array}$ & .28571 & .67302 & .10385 & 2.751 & 41 & .009 \\
\hline Pair 7 & $\begin{array}{l}\text { Attendance-Grad-Level } \\
\text { Attendance-Under-Level }\end{array}$ & -.41667 & .87423 & .14571 & -2.860 & 35 & .007 \\
\hline Pair 8 & $\begin{array}{l}\text { Attendance-Grad-Value } \\
\text { Attendance-Under-Value }\end{array}$ & -.19355 & .54279 & .09749 & -1.985 & 30 & .056 \\
\hline Pair 9 & $\begin{array}{l}\text { Reseach Proj.-Grad-Level } \\
\text { Research Proj.-Grad-Value }\end{array}$ & -.26190 & .58683 & .09055 & -2.892 & 41 & .006 \\
\hline Pair 10 & $\begin{array}{l}\text { Research Proj.-Under-Level } \\
\text { Research Proj.-Under-Value }\end{array}$ & -.38095 & 1.03482 & .15968 & -2.386 & 41 & .022 \\
\hline Pair 11 & $\begin{array}{l}\text { Reseach Proj.-Grad-Level } \\
\text { Research Proj.-Under-Level }\end{array}$ & .40541 & .83198 & .13678 & 2.964 & 36 & .005 \\
\hline Pair 12 & $\begin{array}{l}\text { Research Proj.-Grad-Value } \\
\text { Research Proj.-Under-Value }\end{array}$ & .36667 & .71840 & .13116 & 2.796 & 29 & .009 \\
\hline Pair 13 & $\begin{array}{l}\text { Guest Lecturers-Grad-Level } \\
\text { Guest Lecturers-Grad-Value }\end{array}$ & -.96970 & 1.21153 & .21090 & -4.598 & 32 & .000 \\
\hline Pair 14 & $\begin{array}{l}\text { Guest Lecturers-Under-Level } \\
\text { Guest Lecturers-Under-Value }\end{array}$ & -.87097 & 1.08756 & .19533 & -4.459 & 30 & .000 \\
\hline Pair 15 & $\begin{array}{l}\text { Guest Lecturers-Grad-Level } \\
\text { Guest Lecturers-Under-Level }\end{array}$ & .02703 & .37167 & .06110 & .442 & 36 & .661 \\
\hline Pair 16 & $\begin{array}{l}\text { Guest Lecturers-Grad-Value } \\
\text { Guest Lecturers-Under-Value }\end{array}$ & .04348 & .56232 & .11725 & .371 & 22 & .714 \\
\hline Pair 17 & $\begin{array}{l}\text { Teams-Grad-Level } \\
\text { Teams-Grad-Value }\end{array}$ & .02381 & .74860 & .11551 & .206 & 41 & .838 \\
\hline Pair 18 & $\begin{array}{l}\text { Teams-Under-Level } \\
\text { Teams-Under-Value }\end{array}$ & -.09524 & .72615 & .11205 & -.850 & 41 & .400 \\
\hline Pair 19 & $\begin{array}{l}\text { Teams-Grad-Level } \\
\text { Teams-Under-Level }\end{array}$ & .27027 & .73214 & .12036 & 2.245 & 36 & .031 \\
\hline Pair 20 & $\begin{array}{l}\text { Teams-Grad-Value } \\
\text { Teams-Under-Value }\end{array}$ & .21875 & .49084 & .08677 & 2.521 & 31 & .017 \\
\hline
\end{tabular}

The overall paired sample t-tests for hard technologies are presented in Table 3. The number of comparisons in this table is large and, again, we will pick several of interest to briefly discuss. First, pairs 5 through 8 cover the use and value of e-mail. Confirming our visual inspection of the means, e-mail use and value are not significantly different. Moreover, there are no differences between the undergraduate and graduate programs. E-mail faces no constraints, as our inboxes often testify. Second, pairs 13 through 16 address internet access. There are statistically significant gaps at both the graduate level $(p=.006)$ and undergraduate level $(p=.001)$ with the value of internet use 
significantly higher than its level of use. We suggest that this gap is related to the classroom gap that will be discussed below. Third, pairs 17 through 21 address the use of BlackBoard. Interestingly, the levels and values of use are not significantly different. There is also not a significant difference in undergraduate versus graduate levels or values. Since BlackBoard is internet dependent, both the perceived gap in internet access and classroom accessibility to technology may dampen the perceived value of BlackBoard and retard its serious consideration by faculty members. There is also a policy dimension that will be discussed below. Fourth, consider pairs 33 and 34. A value-use gap exists with the value and levels of use of laptops. Laptops are an integral part of the architecture of many technology adapted classrooms. It is not surprising that this gap would exist, as technology deployment has focused on desktop machines. We expect that policy decisions will include the increased use of laptops. Although other comparisons exist, coverage of the selections displays the kinds of questions and issues that will be potentially helpful to both faculty member s and policy makers.

Table 3

Paired Sample t Tests

Hard Technologies

\begin{tabular}{|c|c|c|c|c|c|c|c|}
\hline & & Mean & $\begin{array}{c}\text { Std. } \\
\text { Deviation }\end{array}$ & $\begin{array}{l}\text { Std. Error } \\
\text { Mean }\end{array}$ & $\mathrm{t}$ & $\mathrm{df}$ & $\begin{array}{l}\text { Sig. (2- } \\
\text { tailed) }\end{array}$ \\
\hline Pair 1 & $\begin{array}{l}\text { OH Proj.-Grad-Level } \\
\text { OH Proj.-Grad-Value }\end{array}$ & -.34146 & .88345 & .13797 & -2.475 & 40 & .018 \\
\hline Pair 2 & $\begin{array}{l}\text { OH Proj.-Under-Level } \\
\text { OH Proj.-Under-Value }\end{array}$ & -.46154 & .94162 & .15078 & -3.061 & 38 & .004 \\
\hline Pair 3 & $\begin{array}{l}\text { OH Proj.-Grad-Level } \\
\text { OH Proj.-Under-Level }\end{array}$ & -.08108 & .36350 & .05976 & -1.357 & 36 & .183 \\
\hline Pair 4 & $\begin{array}{l}\text { OH Proj.-Grad-Value } \\
\text { OH Proj.-Under-Value }\end{array}$ & -.06250 & .24593 & .04348 & -1.438 & 31 & .161 \\
\hline Pair 5 & $\begin{array}{l}\text { E-Mail-Grad-Level E-Mail-Grad- } \\
\text { Value }\end{array}$ & -.09524 & .75900 & .11712 & -.813 & 41 & .421 \\
\hline Pair 6 & $\begin{array}{l}\text { E-Mail-Under-Level E-Mail- } \\
\text { Under-Value }\end{array}$ & -.13333 & 91949 & .13707 & -.973 & 44 & .336 \\
\hline Pair 7 & $\begin{array}{l}\text { E-Mail-Grad-Level E-Mail-Under- } \\
\text { Level }\end{array}$ & .08571 & .44533 & .07527 & 1.139 & 34 & .263 \\
\hline Pair 8 & $\begin{array}{l}\text { E-Mail-Grad-Value E-Mail-Under- } \\
\text { Value }\end{array}$ & .03030 & .58549 & .10192 & .297 & 32 & .768 \\
\hline Pair 9 & $\begin{array}{l}\text { Streaming Video-Grad-Level } \\
\text { Streaming Video-Grad-Value }\end{array}$ & -.78261 & .79524 & .16582 & -4.720 & 22 & .000 \\
\hline Pair 10 & $\begin{array}{l}\text { Streaming Video-Under-Level } \\
\text { Streaming Video-Under-Value }\end{array}$ & -.50000 & 1.10335 & .22522 & -2.220 & 23 & .037 \\
\hline Pair 11 & $\begin{array}{l}\text { Streaming Video-Grad-Level - } \\
\text { Streaming Video-Under-Level }\end{array}$ & .05882 & .60006 & .10291 & .572 & 33 & .571 \\
\hline Pair 12 & $\begin{array}{l}\text { Streaming Video-Grad-Value - } \\
\text { Streaming Video-Under-Value }\end{array}$ & -.05556 & .23570 & .05556 & -1.000 & 17 & .331 \\
\hline Pair 13 & $\begin{array}{l}\text { Internet Access-Grad-Level } \\
\text { Internet Access-Grad-Value }\end{array}$ & -.38889 & .80277 & .13380 & -2.907 & 35 & .006 \\
\hline Pair 14 & $\begin{array}{l}\text { Internet Access-Under-Level } \\
\text { Internet Access-Under-Value }\end{array}$ & -.52778 & .90982 & .15164 & -3.481 & 35 & .001 \\
\hline Pair 15 & $\begin{array}{l}\text { Internet Access-Grad-Level } \\
\text { Internet Access-Under-Level }\end{array}$ & .11429 & .67612 & .11429 & 1.000 & 34 & .324 \\
\hline Pair 16 & $\begin{array}{l}\text { Internet Access-Grad-Value } \\
\text { Internet Access-Under-Value }\end{array}$ & -.03704 & .33758 & .06497 & -.570 & 26 & .574 \\
\hline Pair 17 & $\begin{array}{l}\text { BlackBoard-Grad-Level } \\
\text { BlackBoard-Grad-Value }\end{array}$ & -.11429 & .83213 & .14066 & -.813 & 34 & .422 \\
\hline Pair 18 & $\begin{array}{l}\text { BlackBoard-Under-Level } \\
\text { BlackBoard-Under-Value }\end{array}$ & -.06061 & .49620 & .08638 & -.702 & 32 & .488 \\
\hline Pair 19 & $\begin{array}{l}\text { BlackBoard-Grad-Level - } \\
\text { BlackBoard-Under-Level }\end{array}$ & .00000 & .69631 & .11942 & .000 & 33 & 1.000 \\
\hline Pair 20 & BlackBoard-Grad-Value & .00000 & - & - & - & - & - \\
\hline
\end{tabular}




\begin{tabular}{|c|c|c|c|c|c|c|c|}
\hline Pair 21 & $\begin{array}{l}\text { BlackBoard-Under-Value } \\
\text { PowerPoint-Grad-Level } \\
\text { PowerPoint-Grad-Value }\end{array}$ & -.20000 & .79097 & .12506 & -1.599 & 39 & .118 \\
\hline Pair 22 & $\begin{array}{l}\text { PowerPoint-Under-Level } \\
\text { PowerPoint-Under-Value }\end{array}$ & -.15385 & .77929 & .12479 & -1.233 & 38 & .225 \\
\hline Pair 23 & $\begin{array}{l}\text { PowerPoint-Grad-Level } \\
\text { PowerPoint-Under-Level }\end{array}$ & .05714 & .23550 & .03981 & 1.435 & 34 & .160 \\
\hline Pair 24 & $\begin{array}{l}\text { PowerPoint-Grad-Value } \\
\text { PowerPoint-Under-Value }\end{array}$ & .03226 & .31452 & .05649 & .571 & 30 & .572 \\
\hline Pair 25 & $\begin{array}{l}\text { Publishers Aids-Grad-Level } \\
\text { Publishers Aids-Grad-Value }\end{array}$ & -.40476 & .76699 & .11835 & -3.420 & 41 & .001 \\
\hline Pair 26 & $\begin{array}{l}\text { Publishers Aids-Under-Level } \\
\text { Publishers Aids-Under-Value }\end{array}$ & -.30000 & .68687 & .10860 & -2.762 & 39 & .009 \\
\hline Pair 27 & $\begin{array}{l}\text { Publishers Aids-Grad-Level } \\
\text { Publishers Aids-Under-Level }\end{array}$ & -.08571 & .44533 & .07527 & -1.139 & 34 & .263 \\
\hline Pair 28 & $\begin{array}{l}\text { Publishers Aids-Grad-Value } \\
\text { Publishers Aids-Under-Value }\end{array}$ & -.06452 & .24973 & .04485 & -1.438 & 30 & .161 \\
\hline Pair 29 & $\begin{array}{l}\text { Laser Pointer-Grad-Level } \\
\text { Laser Pointer-Grad-Value }\end{array}$ & -.40000 & .82078 & .18353 & -2.179 & 19 & .042 \\
\hline Pair 30 & $\begin{array}{l}\text { Laser Pointer-Under-Level } \\
\text { Laser Pointer-Under-Value }\end{array}$ & .00000 & .53452 & .13801 & .000 & 14 & 1.000 \\
\hline Pair 31 & $\begin{array}{l}\text { Laser Pointer-Grad-Level } \\
\text { Laser Pointer-Under-Level }\end{array}$ & -.08824 & .51450 & .08824 & -1.000 & 33 & .325 \\
\hline Pair 32 & $\begin{array}{l}\text { Laser Pointer-Grad-Value } \\
\text { Laser Pointer-Under-Value }\end{array}$ & -.07143 & .26726 & .07143 & -1.000 & 13 & .336 \\
\hline Pair 33 & $\begin{array}{l}\text { Laptop-Grad-Level Laptop-Grad- } \\
\text { Value }\end{array}$ & -.35484 & .75491 & .13559 & -2.617 & 30 & .014 \\
\hline Pair 34 & $\begin{array}{l}\text { Laptop-Under-Level Laptop- } \\
\text { Under-Value }\end{array}$ & -.35484 & .70938 & .12741 & -2.785 & 30 & .009 \\
\hline Pair 35 & $\begin{array}{l}\text { Laptop-Grad-Level Laptop-Under- } \\
\text { Level }\end{array}$ & .02778 & .16667 & .02778 & 1.000 & 35 & .324 \\
\hline Pair 36 & $\begin{array}{l}\text { Laptop-Grad-Value Laptop-Under- } \\
\text { Value }\end{array}$ & .04167 & .35864 & .07321 & .569 & 23 & .575 \\
\hline Pair 37 & $\begin{array}{l}\text { Elmo Projector-Grad-Level } \\
\text { Elmo Projector-Grad-Value }\end{array}$ & -.21739 & .59974 & .12505 & -1.738 & 22 & .096 \\
\hline Pair 38 & $\begin{array}{l}\text { Elmo Projector-Under-Level Elmo } \\
\text { Projector-Under-Value }\end{array}$ & -.41176 & .79521 & .19287 & -2.135 & 16 & .049 \\
\hline Pair 39 & $\begin{array}{l}\text { Elmo Projector-Grad-Level Elmo } \\
\text { Projector-Under-Level }\end{array}$ & .02941 & .17150 & .02941 & 1.000 & 33 & .325 \\
\hline Pair 40 & $\begin{array}{l}\text { Elmo Projector-Grad-Value Elmo } \\
\text { Projector-Under-Value }\end{array}$ & -.06667 & .25820 & .06667 & -1.000 & 14 & .334 \\
\hline Pair 41 & $\begin{array}{l}\text { Infocus Projector-Grad-Level } \\
\text { Infocus Projector-Grad-Value }\end{array}$ & -.30000 & .57124 & .12773 & -2.349 & 19 & .030 \\
\hline Pair 42 & $\begin{array}{l}\text { Infocus Projector-Under-Level } \\
\text { Infocus Projector-Under-Value }\end{array}$ & -.25000 & .57735 & .14434 & -1.732 & 15 & .104 \\
\hline Pair 43 & $\begin{array}{l}\text { Infocus Projector-Grad-Level } \\
\text { Infocus Projector-Under-Level }\end{array}$ & -.09091 & .72300 & .12586 & -.722 & 32 & .475 \\
\hline Pair 44 & $\begin{array}{l}\text { Infocus Projector-Grad-Value } \\
\text { Infocus Projector-Under-Value }\end{array}$ & .14286 & .53452 & .14286 & 1.000 & 13 & .336 \\
\hline
\end{tabular}

The overall paired sample t-tests for classroom types are presented in Table 4. The message here is that the value of smart or hybrid classrooms is significantly greater than the level of their use, and that the level of use of standard classrooms is greater than their perceived value. We view this as the major constraint surrounding the valueuse gap of a variety of hard technologies. 
Table 4

Paired Sample t Tests

Classroom Technologies

\begin{tabular}{|c|c|c|c|c|c|c|c|}
\hline & & Mean & $\begin{array}{l}\text { Std. } \\
\text { Deviation }\end{array}$ & $\begin{array}{l}\text { Std. Error } \\
\text { Mean }\end{array}$ & $\mathrm{t}$ & df & $\begin{array}{l}\text { Sig. (2- } \\
\text { tailed) }\end{array}$ \\
\hline Pair 1 & $\begin{array}{l}\text { Smart Classroom-Grad-Level } \\
\text { Smart Classroom-Grad-Value }\end{array}$ & -.51163 & .76756 & . 11705 & -4.371 & 42 & .000 \\
\hline Pair 2 & $\begin{array}{l}\text { Smart Classroom-Under-Level } \\
\text { Smart Classroom-Under-Value }\end{array}$ & -.55000 & .87560 & .13844 & -3.973 & 39 & .000 \\
\hline Pair 3 & $\begin{array}{l}\text { Smart Classroom-Grad-Level } \\
\text { Smart Classroom-Under-Level }\end{array}$ & .08571 & .56211 & .09501 & .902 & 34 & .373 \\
\hline Pair 5 & $\begin{array}{l}\text { Hybrid Classroom-Grad-Level } \\
\text { Hybrid Classroom-Grad-Value }\end{array}$ & -.65517 & .97379 & 18083 & -3.623 & 28 & .001 \\
\hline Pair 6 & $\begin{array}{l}\text { Hybrid Classroom-Under-Level - } \\
\text { Hybrid Classroom-Under-Value }\end{array}$ & -.72727 & 1.03901 & .18087 & -4.021 & 32 & .000 \\
\hline Pair 7 & $\begin{array}{l}\text { Hybrid Classroom-Grad-Level } \\
\text { Hybrid Classroom-Under-Level }\end{array}$ & 12121 & .54530 & .09492 & 1.277 & 32 & 211 \\
\hline Pair 8 & $\begin{array}{l}\text { Hybrid Classroom-Grad-Value } \\
\text { Hybrid Classroom-Under-Value }\end{array}$ & .00000 & .28284 & .05547 & . 000 & 25 & 1.000 \\
\hline Pair 9 & $\begin{array}{l}\text { Standard Classroom-Grad-Level } \\
\text { Standard Classroom-Grad-Value }\end{array}$ & .38889 & .72812 & .12135 & 3.205 & 35 & .003 \\
\hline $\begin{array}{l}\text { Pair } \\
10\end{array}$ & $\begin{array}{l}\text { Standard Classroom-Under-Level } \\
\text { Standard Classroom-Under-Value }\end{array}$ & .45946 & 69100 & 11360 & 4.045 & 36 & .000 \\
\hline $\begin{array}{l}\text { Pair } \\
11\end{array}$ & $\begin{array}{l}\text { Standard Classroom-Grad-Level } \\
\text { Standard Classroom-Under-Level }\end{array}$ & -.10000 & .30513 & .05571 & -1.795 & 29 & .083 \\
\hline $\begin{array}{l}\text { Pair } \\
12\end{array}$ & $\begin{array}{l}\text { Standard Classroom-Grad-Value } \\
\text { Standard Classroom-Under-Value }\end{array}$ & .10345 & .55709 & .10345 & 1.000 & 28 & .326 \\
\hline
\end{tabular}

Since we tracked factors for teaching experience, status, and departmental affiliation, we next investigated whether or not these factors were significant within the context of the overall results. We ran a univariate ANOVA on the response to each variable with a model including the three factors. If a factor was significant, we ran both Least Significant Difference (LSD) and Bonferroni post hoc tests to determine the nature and direction of the difference. Table 5 illustrates the significant findings at .05 using LSD with the supplemental Bonferroni measure displayed. The Bonferroni test adjusts for the number of tests being made. So, there were fewer tests that survived both comparisons. The most significant results are the several that are reported as significant under both tests.

The differences we examine here should be understood in the context that the factors were non-significant in examining the means of most measures. Only a minority of measures scored as significant. Those that survived both the LSD and more restrictive Bonferroni test were fewer still. Table 5 indicates that ten technologies involving twelve factors scored as significant. Of those, six were related to status, two involved experience level, and four involved department affiliation. We will focus our discussion on the scores involving status as those scores were 1) the most likely to survive both post hoc tests and suggest a high level of significance, and 2) the scores directly involve the most recently included new faculty.

New faculty strongly tended to use $\mathrm{OH}$ projectors, Internet Access, BlackBoard, and Laptops at rates significantly higher than other full time and part-time faculty. Since the addition of new faculty has been large enough to constitute a significant percentage of full-time faculty members, the general level of use and demand for facilities noted previously will tend to rise. The influx of new faculty combined with policy initiatives and training that includes full time as well as part-time faculty may significantly raise the overall levels of technology adoption across the school of business.

Figure 5 shows selected graphs of estimated marginal means of the scores discussed above charted against either teaching experience or teaching field. The horizontal axis is coded for status where " $\mathrm{F}$ " = full time, "N" = new, and "P" = part time faculty members. Panels A through F show the clear differences across the faculty groups. 
Table 5

Post Hoc Test Results

\begin{tabular}{|c|c|c|c|c|c|c|c|}
\hline Technology & $\begin{array}{l}\text { Undergrad (U) } \\
\text { Graduate }(\mathrm{G})\end{array}$ & Use Type & $\begin{array}{l}\text { Factor } \\
\text { Name }\end{array}$ & $\begin{array}{l}\text { Factor } \\
\text { Sig. }\end{array}$ & $\begin{array}{l}\text { Post Hoc } \\
\text { LSD }\end{array}$ & $\begin{array}{l}\text { Post Hoc } \\
\text { Bonferro } \\
\text { ni }\end{array}$ & $\begin{array}{l}\text { Direction of } \\
\text { Result }\end{array}$ \\
\hline \multirow[t]{2}{*}{ OH Projector } & $\mathrm{U}$ & Level & Status & .022 & .009 & .028 & New $>$ Full \\
\hline & & & & & .007 & .021 & New $>$ Part \\
\hline \multirow[t]{2}{*}{ OH Projector } & $\mathrm{U}$ & Value & Exp. & .037 & .032 & .096 & $<4>4-12$ \\
\hline & & & & & .049 & .147 & $>12>4-12$ \\
\hline \multirow[t]{2}{*}{ E-Mail } & $\mathrm{U}$ & Level & Field & .046 & .007 & .072 & Acc $>$ Mkt \\
\hline & & & & & .040 & .398 & Fin $>$ Mkt \\
\hline \multirow[t]{2}{*}{$\begin{array}{l}\text { Internet } \\
\text { Access }\end{array}$} & G & Level & Status & .020 & .005 & .014 & New $>$ Full \\
\hline & & & & & .001 & .012 & New $>$ Part \\
\hline \multirow[t]{3}{*}{ BlackBoard } & $\mathrm{G}$ & Level & Field & .018 & .034 & .336 & Acc $>$ Mkt \\
\hline & & & & & .024 & .236 & Acc $>$ Fin \\
\hline & & & & & .037 & .372 & Mkt $>$ Fin \\
\hline \multirow[t]{2}{*}{ BlackBoard } & $\mathrm{U}$ & Level & Status & .026 & .001 & .002 & New $>$ Full \\
\hline & & & & & .000 & .001 & New > Part \\
\hline \multirow{2}{*}{ Laptop } & $\mathrm{U}$ & Level & Status & .023 & .000 & .001 & New $>$ Full \\
\hline & & & & & .005 & .015 & New $>$ Part \\
\hline \multirow[t]{4}{*}{$\begin{array}{l}\text { Guest } \\
\text { Lecturers } \\
\end{array}$} & $\mathrm{U}$ & Level & Exp. & .031 & .029 & .086 & $4-12>>12$ \\
\hline & & & Field & .028 & .050 & .497 & Mkt $>$ MIS/Ops \\
\hline & & & & & .010 & .102 & Mgmt $>$ Acc \\
\hline & & & & & .023 & .228 & $\begin{array}{l}\text { Mgmt > } \\
\text { MIS/ops }\end{array}$ \\
\hline \multirow[t]{4}{*}{$\begin{array}{l}\text { Guest } \\
\text { Lecturers }\end{array}$} & $\mathrm{U}$ & Value & Status & .031 & .051 & .152 & Full > Part \\
\hline & & & Field & .016 & .040 & .396 & Fin $>$ MIS/ops \\
\hline & & & & & .029 & .288 & Mkt $>$ Acc \\
\hline & & & & & .012 & .117 & Mkt > MIS/ops \\
\hline \multirow[t]{2}{*}{ Teams } & $\mathrm{G}$ & Value & Status & .035 & .009 & .027 & Full > Part \\
\hline & & & & & .010 & .03 & New > Part \\
\hline
\end{tabular}

Figure 5

Selected Plots of Results

Panel A

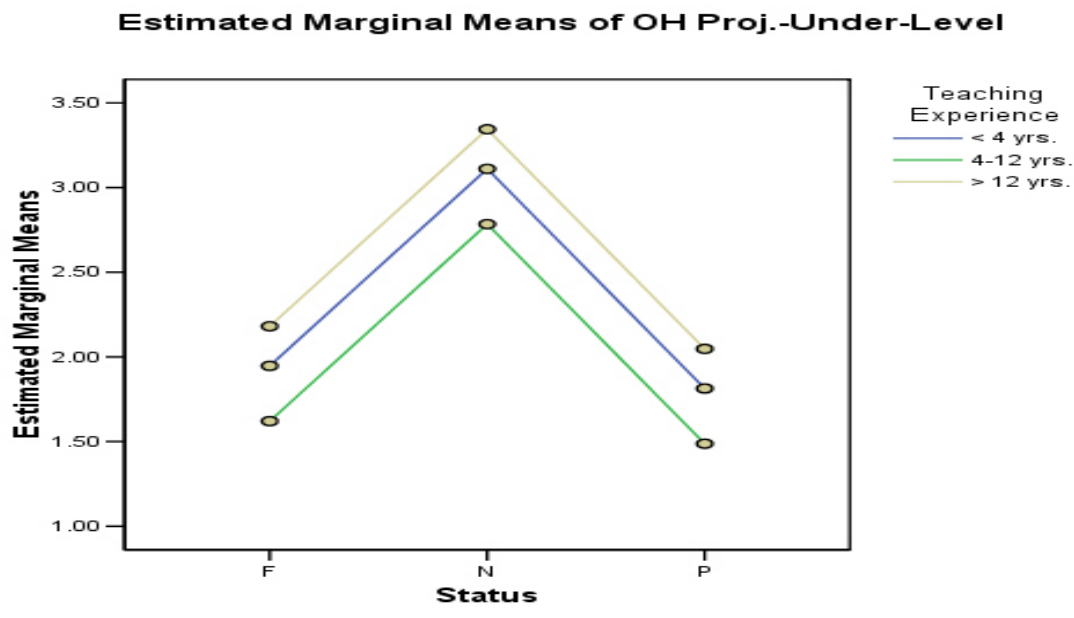


Figure 5 - Continued

Panel B

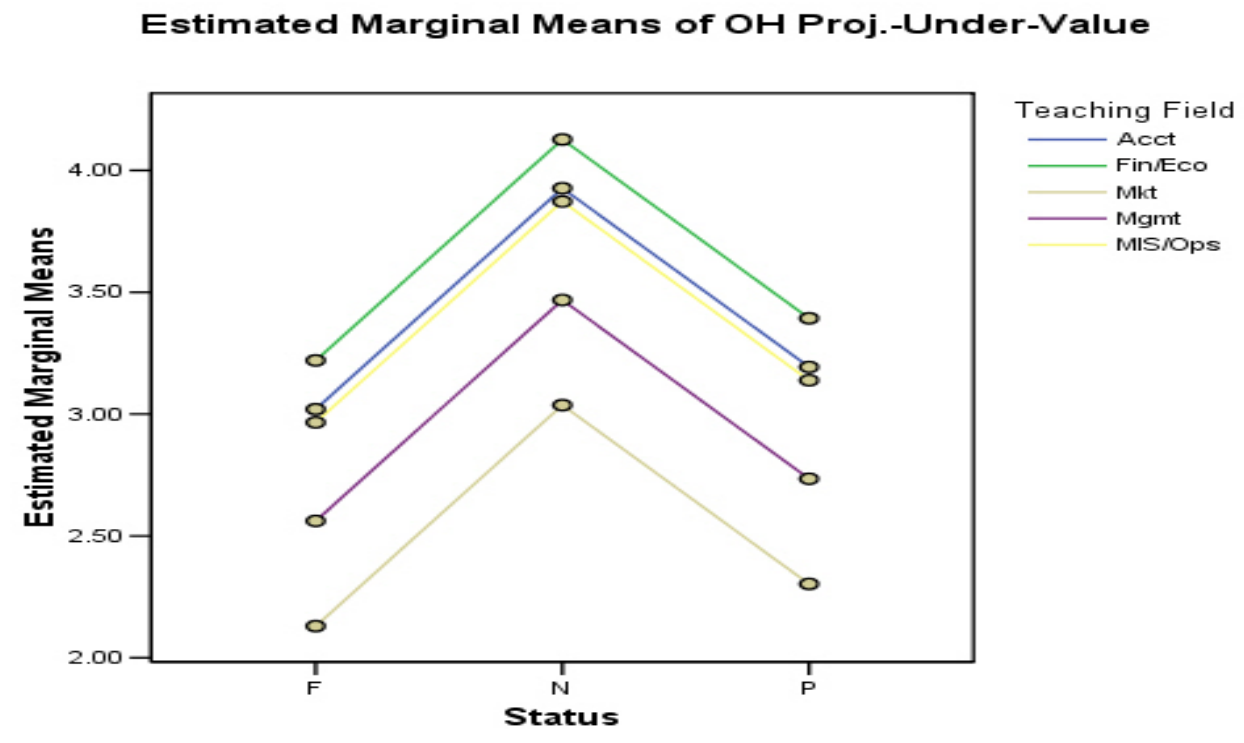

Panel C

Estimated Marginal Means of Laptop-Grad-Level

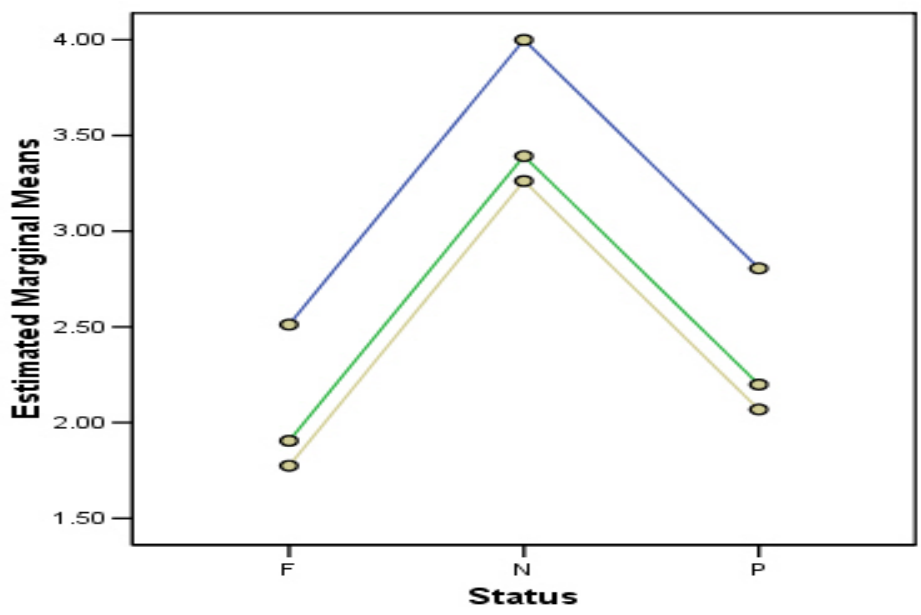

Teaching Experience $<4$ yrs. - 4-12 yrs. $>12$ yrs. 
Figure 5 - Continued Panel D

\section{Estimated Marginal Means of Internet Access-Under-Level}

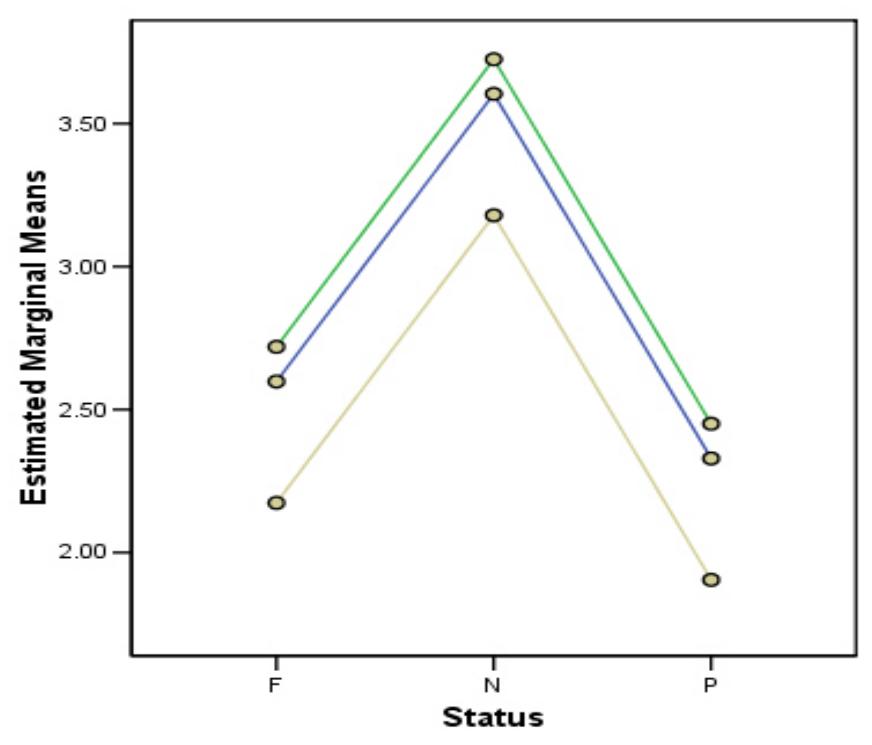

$$
\begin{gathered}
\begin{array}{c}
\text { Teaching } \\
\text { Experience }
\end{array} \\
\begin{array}{r}
<4 \mathrm{yrs} . \\
4-12 \mathrm{yrs} . \\
>12 \mathrm{yrs} .
\end{array}
\end{gathered}
$$

\section{Estimated Marginal Means of BlackBoard-Grad-Level}

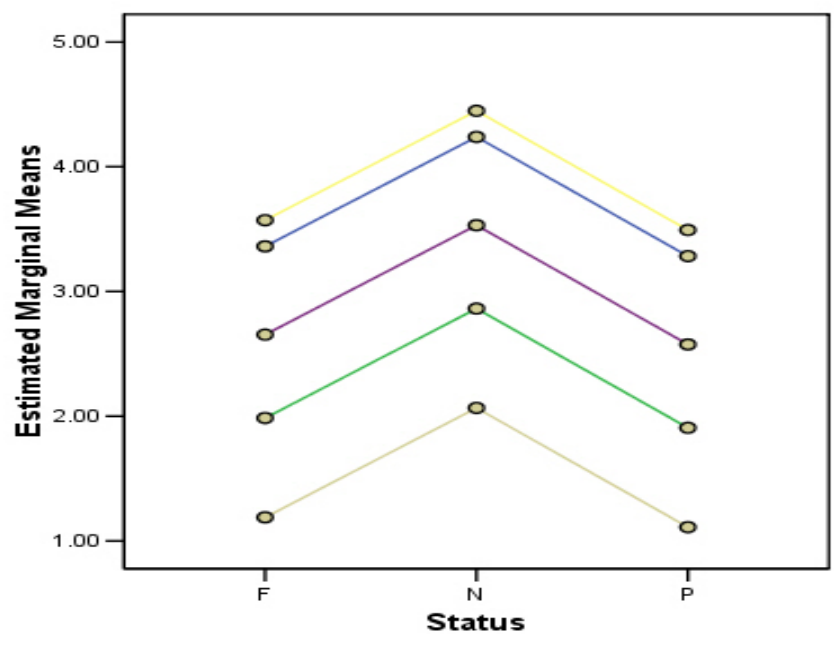

$$
\begin{aligned}
& \text { Teaching Field } \\
& \text { - Acct } \\
& \text { Mkt } \\
& \text { MIslops }
\end{aligned}
$$




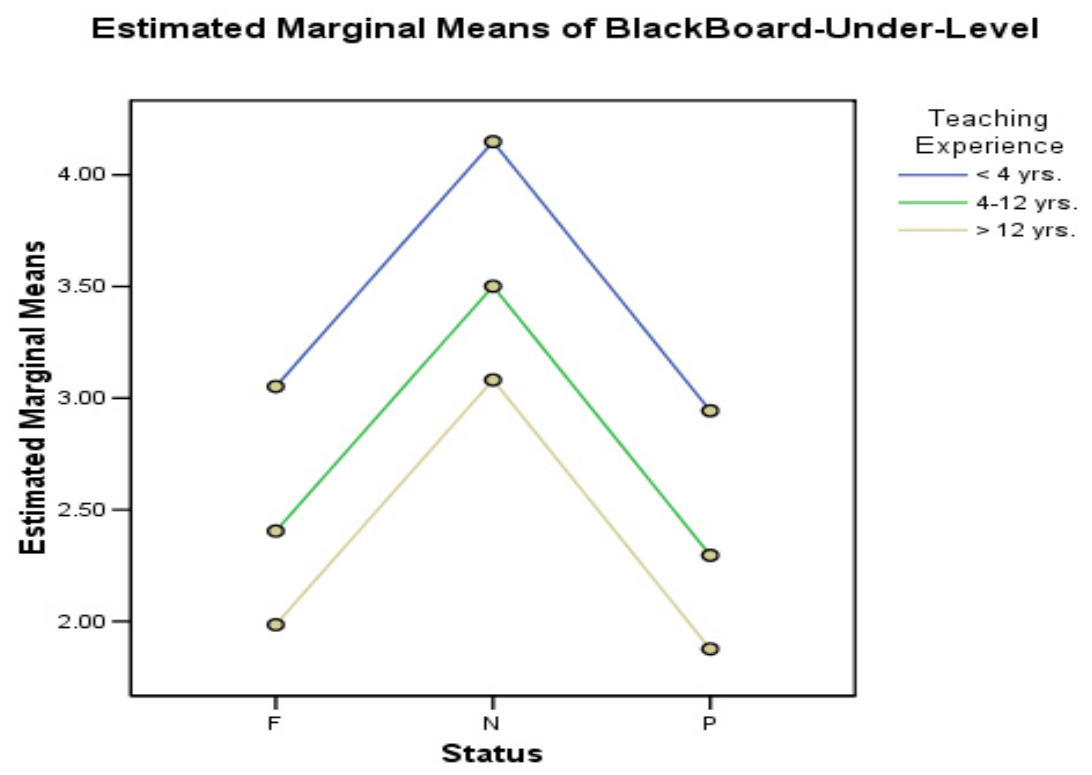

\section{LIMITATIONS AND DELIMITATIONS}

The research findings are delimited to the faculty at the one school studied. The results may not be generalized. It is our hope that such an approach could, however, be applied at other schools individually as case studies, and in larger studies to observe patterns across different settings. We are also delimited by the sample size and the number of tests from establishing stronger links in post hoc tests. We graphed many of the factor differences and those will be presented to the institution for consideration as part of the AACSB-I continuous improvement process. The study is also delimited to descriptions and comparisons of the values and use of various technologies without attempting development of either correlation or causal links to major outcome measures linked to teaching satisfaction, teaching quality, or student learning $(41,42)$.

\section{DISCUSSION, CONCLUSIONS AND RECOMMENDATIONS}

With respect to differences between value and use of the various methods and technologies, we found many statistically significant differences between the perceived value and level of use of many of the soft technologies, many of the hard technologies, and a clear gap between the value and level of use for technologically equipped classroom types. A visual comparison of the means and the $t$ tests indicate that in general, many of the technologies scored higher in value than in level of use and that many of the differences were statistically significant.

The findings have policy implications for both faculty and administrators. Identification and consideration of the constraints as perceived by faculty is an interesting next step. One of the major constraints in the current setting may be the progressive build out of smart or hybrid classrooms. Access by an increasing number of faculty members to the improved classroom resources may elevate many other constraints such as use of laptops or streaming video. Even the use of a laser pointer might be affected by both technology and classroom configuration. Other constraints may have to do with a need for additional training and/or standardization. For example, BlackBoard is available and recommended for use. However, it is not required and all elements of BlackBoard may not be familiar to faculty. 
Group management features are available in BlackBoard that includes e-mail as well as other tools. We know that faculty both highly value and intensely use e-mail. It is possible that faculty would be amenable to implementing a course management tool like BlackBoard as standard if use is clearly linked to the types of technologies such as email communications and teams that they may value highly. In effect, we recommend a debriefing and analysis of the overall findings as we believe that the results of such a debriefing may inform change and encourage progress. There are several sources available to further investigate BlackBoard or Course Management software generally $(5,25,37$, and 38).

Departments were generally not significant in explaining differences in values or levels of use of most technologies. Exceptions were found in the "Value of Guest Speakers" where, somewhat expectedly, Marketing and Management scored significantly higher than Operations and Accounting. The "Value of Teams" was also less important to Operations than to Management. Is collaborative learning equally effective across all disciplines or should there be expected differences in the use of teams based on course content (15)?

Status tracked as full time, new, and adjunct was also not a significant factor on most dimensions. Adjunct faculty tended to see higher value to Attendance and lesser value to Research Projects and to Team Projects at the graduate level. However, it is interesting that new faculty scored significantly higher on a cluster of hard technologies than continuing faculty. The increase in demand for technology may further spur demand for compatible classrooms, and the increased number of technology users may aid in the diffusion of technology innovation $(18,33)$.

Teaching Experience tended to be non-significant across almost all dimensions. However, the level of use of internet access was significantly lower at the undergraduate level for experience levels greater than twelve years. This finding may suggest an opportunity for increasing the comfort level and communicating the potential benefits of internet usage to highly experienced faculty.

Future research might replicate additional case studies at other universities. Especially relevant may be business schools entering a period of self study or re-accreditation. Our research introduces a structured approach to data collection and analysis for reviewing the way faculty as a whole use technology; identification of the gaps between use and value of a technology; comparison of graduate and undergraduate differences in techniques; and variation across groups of faculty and faculty departments. A wide variety of statistical comparisons and graphic illustration may help focus renewal and development efforts.

An interesting application of the instrument and analysis within the context of AACSB-I would be to test a self-identified peer group to observe similarities and differences across the dimensions. The larger research questions related to teaching satisfaction, teaching quality, and student learning linked to technology choice and use intensity is arguably the most interesting extension. We may find that simple expansion of technology use is not so clearly linked to learning outcomes (7). There may exist clusters of approaches and technologies, coupled with dimensions not captured in this study, that most clearly lead to desired outcomes (24).

Once issues are identified, goals are established, and policies are set, institutions need effective and extensive support systems to be available to support change. Various institutions have established a variety of standards and programs to nurture change, improve effectiveness, and reduce resistance $(1,2,11,22,27$, and 28).

\section{ACKNOWLEDGEMENTS}

Special appreciation is extended to Dean Anthony F. Libertella for his initial encouragement to pursue the research and his subsequent support for presentation of the papers at four U.S. conferences and for presentation at a conference at Oxford University. We owe additional thanks to the faculty at Adelphi for their ongoing participation. Finally, we thank the attendees at our previous paper presentations for their constructive feedback and encouragement. 


\section{REFERENCES}

1. AACSB International, Eligibility Procedures and Accreditation Standards for Business Accreditation, Tampa, Florida, Revised: January 01, 2005, p.35-37.

2. Adelphi University Faculty Center for Professional Excellence, http://fcpe.adelphi.edu.

3. Arabasz, Paul and Baker, Mary Beth, Evolving Campus Support Models for E-learning Courses, EDUCAUSE Center for Applied Research, Boulder, Colorado, March 2003.

4. Bianco-Mathis Virginia and Chalofsky eds., The Adjunct Faculty Handbook, Sage Publication, 1996, p.55.

5. Blackboard, http://www.blackboard.com/about/index.htm

6. Bowen, Howard R. and Schuster, Jack H., American Professors: A National Resource Imperiled; Oxford University Press, 1986, p.60-66.

7. Caruso, Judith Borreson, Educause Center for Applied Research ECAR, Study of Students and Information Technology, 2004: Convenience, Connection and Control, Boulder, Colorado.

8. Carroll, Jill, Do Adjuncts Have Time for Students? Chronicle of Higher Education; 7/25/2003, Vol. 49 Issue 46, P.C 4.

9. $\quad$ Chronicle of Higher Education Speaker says Adjuncts May Harm Students, November 18, 2005, p.13.

10. Conley, Valerie Martin and Leslie David W., Part-time Instructional faculty and Staff: Who They Are, What they do and what they think, NCES 2002-163, p.8.

11. Dalhousie University's Instructional Development and Technology Sites Worldwide, Office of Instructional Development and Technology: http://www.dal.ca/noidt/ids.html

12. Ehrenberg, Ronald G., The Changing Nature of the Faculty Employment Practices Revised Draft, November 6, 2005, Presented at the TIAA-CREF Institute Conference on "The New Balancing Act in Higher Education,” NY, November 3-5, 2005. Retrieved from www.TIAA-CREFInstitute.org on 11/21/05.

13. Forrest Cataldi, E., Fahimi, M., and Bradburn, E.M. (2005). 2004 National Study of Postsecondary Faculty (NSOPF: 04) Report on Faculty and Instructional Staff in Fall 2003 (NCES 2005-172). U.S. Department of Education. Washington, DC: National Center for Education Statistics. Retrieved from http://nces.ed.gov/pubsearch.

14. Fulton, Richard D., The Plight of Part-timers in Higher Education. Change; May/ June 2000, Vol. 32, Issue 3, p.6. As retrieved from Adelphi online resources.

15. Gokhale, Anuradha A., Collaborative Learning Enhances Critical Thinking, http://scholar.lib.vt.edu/ejournals/jte/jte-v>n//gokhale.jte-v7n1.html

16. Grappa, Judith M., Part-time Faculty: Higher Education at a Crossroads; Association for the Study of Higher Education, Report 3; 1984, p.4.

17. Grieve, Donald and Warden, Catherine, eds., Handbook II- Advanced Teaching Strategies for Adjunct Faculty

18. Hall, Mark and Elliot, Kevin M., Diffusion of Technology into the teaching process: Strategies to encourage faculty members to embrace the lap top environment, Journal of Education for Business. Washington July/August 2003. Vol.78.

19. http://www.4faculty.org (version4) accessed on 10/19/05.

20. http://www.AdjunctNation.com/ accessed on 11/26/05.

21. http://www.Adjunctopia.com/ accessed on 11/26/05.

22. Knight, Mark 4faculty.org- An Innovative Approach to Faculty Development Community College Journal of Research and Practice; Jan 2004, Vol. 28, Issue 1, p55-56. As retrieved online from Adelphi.edu/servlLet/WeBACC?Action=Item

23. Lyons, Richard E, et al, Adjunct Professor's Guide to Success, The Surviving and Thriving in the College Classroom, Allyn \& Green, $1^{\text {st. }}$. ed., 2003.

24. Ma, Yulong and Runyon, L.R., Academic Synergy in the Age of Technology - A New Instructional Paradigm, Journal of Education for Business. Washington - July/August 2004. Vol. 79.

25. Morgan, Glenda. Faculty Use of Course Management Systems, EDUCAUSE Center for Applied Research (ECAR), Boulder, Co., May 2003.

26. National Education Association Part-time and Temporary Faculty, 1989, p.1-3.

27. Office of Instructional Consultation, University of California at Santa Barbara, http://www.id.ucsb.edu/ic/resources/collab-l/difference.html. 
28. Office of Instructional Consultation, University of California at Santa Barbara, http://www.id.ucsb.edu/ic/resources/collab-l/difference.html.

29. Payette Dennis L., Enhanced Multi-faceted Teaching Methods, Journal of College Teaching and Learning, Vol. 1, No. 6, June 2004, p. 9-16.

30. Payette Dennis L. and Verreault, Daniel, Enhanced Multi-faceted Teaching Methods Phase II, Journal of College Teaching and learning, Vol. 2. No. 6, June 2005, p. 31-45.

31. Payette, D. and Verreault, D., Enhanced Multi-Faceted Teaching Methods: Phase III The Journal of College Teaching and Learning, June, 2006, p. 1-18.

32. Payette, D. and Verreault, D., Mining Faculty Data on Teaching Methods and Technologies A Case Study at the Adelphi University School of Business at the Oxford University 8th International Conference on Social Values in Education and Business, Oxford, England, July 19th--21st, 2006. Presented by D. Payette. Proceedings Forthcoming.

33. $\quad$ Rogers, E.M., Diffusion of Innovation ( $3^{\text {rd }}$ Ed.) New York: The Free Press 1983.

34. Schroeder, Sandra, It's Time to give Adjuncts a Break, Chronicle of higher Education, October 28, 2005, p. B 27.

35. Sonner, Brenda S. A Is for Adjunct Examining Grade Inflation in Higher Education, Journal of Education for Business; Sept/Oct 2000, Vol. 76 Issue 1, p.5 as retrieved online through Adelphi.edu/servlLet/WeBACC.

36. The American Association of University Professors 1940 Statement of Principles on Academic Freedom and Tenure (www.aaup.org/statements, accessed June 5, 2006).

37. Ullman, Craig and Rabinowitz, Mitchell Course Management Systems and the Reinvention of Instruction, T.H.E. Journal Web Exclusive, October 2004, http://thejournal.com/magazine/vault/A5070.cjm

38. University of Wisconsin, Course Management System Survey, EDUCAUSE Center for Applied Research, Vol. 2, 2003.

39. U.S. Department of Education, National center for Education Statistics (2002). A profile of Part-time Faculty: Fall 1998, NCES 2002-08, by Andrea Berger, Rita Kirshstein, Yu Zhang, and Kevin Carter, American Institute for Research, Linda J. Zimbler, Project Officer. Washington, DC: 2002. Accessed 11/26/05 from http://nces.ed.gov/pubsearch.

40. U.S. Department of Education. National Center for Education Statistics. E.D. TABS: Fall Staff in Postsecondary Institutions, 1995, NCES 98-228, by Stephen Roey and Rebecca Rak. Project Officers, Rosa Fernandez and Sam Barbett. Washington, D.C.: 1998, p.1-2. Accessed 11/26/05 from http://nces.ed.gov/pubsearch.

41. Wark, Elizabeth J., Technology as a Tool, Not a Teacher Replacement Letter to the Teaching Professor, Magna Publications, October 2004.

42. Young, Jeffrey R., Students Say Technology has Little Impact on Teaching, Chronicle of Higher Education; 8/13/2004, Vol. 50 Issue 49, PA 28, 1/3 p.

43. Wickun, William G. and Rock, Stanley E., The Role of Adjunct Faculty in Higher Education The Montana Professor Academic Journal http://MTPRof.MSUN.edu/Win2000/Wickun.html. Accessed on 11/26/05. 